Notfall Rettungsmed 2009 · 12:7-8

DOI 10.1007/s10049-008-1110-0

Online publiziert: 21. Januar 2009

(c) Springer Medizin Verlag 2009

\author{
C. Madler ${ }^{1}$ • U. Kreimeier ${ }^{2}$ \\ ${ }^{1}$ Institut für Anaesthesiologie und Notfallmedizin I, Westpfalz- \\ Klinikum GmbH Kaiserslautern, Kaiserslautern \\ ${ }^{2}$ Klinik für Anaesthesiologie, Klinikum der Universität München, München
}

\title{
Die soziale Dimension der Notfallmedizin
}

hin, welche zunehmend die Einsatzrealität gerade auch in Ballungsräumen bestimmen. Soziale Benachteiligung prädestiniert auch dazu, an notfallmedizinischen Tracerdiagnosen wie Myokardinfarkt und Schlaganfall zu erkranken bzw. schwere Verletzungen zu erleiden. Medizinsoziologisches Hintergrundwissen des Notfallteams in der Akutversorgung ist nicht zuletzt auch deshalb von besonderer Bedeutung, weil es unterschiedliche Krankheitseinsicht und unterschiedliches Meldeverhalten von Patienten und Angehörigen erklärt.

\section{Technische Assistenzsysteme sind kein Ersatz für soziale Bindungen}

Im Hinblick auf die zweite große gesellschaftliche Herausforderung, nämlich die demographische Entwicklung, ist die Notfallmedizin aufgerufen, nicht nur durch Anpassung an die individualmedizinischen Bedürfnisse betagter Patienten zu reagieren, sondern auch hinsichtlich der Verpflichtung, neue Versorgungsstrategien mitzugestalten. S. Prückner et al. stellen deshalb nicht nur die demographische Entwicklung und die medizinischen und sozialen Besonderheiten des geriatrischen Notfallpatienten in den Mittelpunkt ihres Beitrags, sie weisen auch auf die Verpflichtung der Notfallmedizin hin, ihre Expertise in die Entwicklung von technischen Assistenzsystemen, die ein sicheres und unabhängiges Leben im Alter ermöglichen sollen, einfließen zu lassen.

Das biopsychosoziale Vitalitätsmodell des Menschen, welches dem Konzept einer elektronischen Notfallerkennung für alleinlebende alte Menschen zugrunde liegt, wird von J. Nehmer ausführlich dargestellt. Sein Beitrag macht deutlich, welchen Stellenwert das Thema ,assisted living" für unsere Arbeit in Zukunft einnehmen wird.

Unsere Aufgabe als Notärzte ist es aber auch, darauf hinzuwirken, dass technische Assistenzsysteme nicht dazu verwendet werden dürfen, soziale Bindungen zu ersetzen, sondern sie zu unterstützen $[4,5]$. Die Inkongruenz zwischen den Bedürfnissen unserer Gesellschaft, den von ihr abgeforderten Gesundheitsleistungen und den traditionellen Versorgungsstrukturen ist in der Notfallmedizin sehr unmittelbar zu spüren. Immer mehr wird der Notarzt zum medico-psychosozialen Libero.

Neben dem sozialen Wandel sind es vor allen Dingen Weiterentwicklungen medizinischer Behandlungsstrategien und technologischer Möglichkeiten sowie ökonomische Vorgaben, die es dringend geboten erscheinen lassen, die traditionellen Versorgungsstrukturen der Akutversorgung zu hinterfragen. C.K. Lackner et al. weisen insbesondere auf die sich abzeichnende Rolle der Notfallmedizin in einer vernetzten regionalen Gesundheitsversorgung hin.

\section{( In der Präklinik erlebt der Arzt unmittelbar die soziale Realität des Patienten}

Zu guter Letzt: Für junge Ärzte ist die präklinische Notfallmedizin eine der letzten verbliebenen Möglichkeiten, die soziale Realität von Patienten unmittelbar zu erleben. Hier erfährt man - im wahrsten Sinne des Wortes - die Bedeutung des 
Kontextes für die Entstehung und Ausprägung akuter Erkrankung und Verletzung. Dies allein wäre Grund genug, sich der sozialen Dimension der Notfallmedizin verstärkt zu widmen.

Medicin ist eine sociale Wissenschaft, und Politik ist weiter nichts als Medicin im Großen. (Zitat aus R. Virchow, 1849: „Die Noth im Spessart")

Diese Maxime Rudolf Virchows gilt bis heute. Und wie wir meinen, für die Notfallmedizin in besonderem Maße.

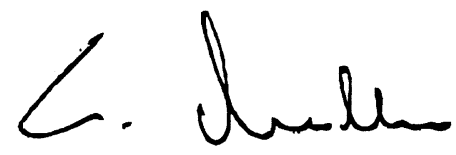

C. Madler

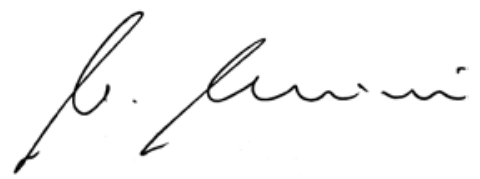

U. Kreimeier

\section{Korrespondenzadresse}

Prof. Dr. C. Madler

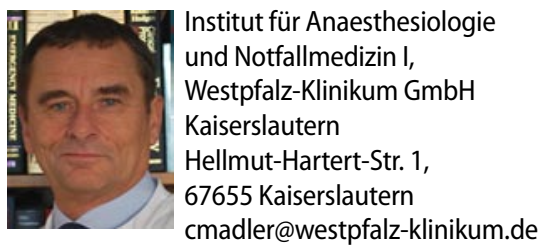

\section{Literatur}

1. Albers L (2002) in: Albers L, Leiß O (Hrsg) Körper - Sprache - Weltbild. Integration biologischer und kultureller Interpretationen in der Medizin. Schattauer, Stuttgart

2. Lüttgen R, Mendel F, Hennes HP (Hrsg) (2005) Bayerisches Rettungsdienstgesetz. Handbuch des Rettungswesens. Mendel, Witten, B 3.205

3. Dick WF (2002) Notarzt oder KV-Arzt? - Die Aufgaben der präklinischen Versorgung. Notfall Rettungsmed 5:572-573

4. Prückner S, Luiz T, Steinbach-Nordmann S et al (2008) Notfallmedizin - Medizin für eine alternde Gesellschaft. Anästhesist 4:391-396

5. Prückner S, Schell B, Luiz T, Madler C (2008) Der Arbeitslose als Notfallpatient. Prospektive Studie zum sozialen Kontext von Notarzteinsätzen. Notfall Rettungsmed 11:557-562

\section{Ambiente Systeme}

Der Forschungsschwerpunkt „AmSys" wurde im Rahmen der Forschungsinitiative des Landes Rheinland-Pfalz im Juni 2008 an der TU Kaiserslautern eingerichtet. Er befasst sich mit Technologie und Anwendung ambienter Systeme.

Unter ambienten Systemen versteht man eine neue Generation eingebetteter Systeme, die weitgehend autonom und bedienungsfrei arbeiten. Ihr Handlungsrahmen wird durch proaktive Beobachtung und Interpretation ihrer Umgebung über Sensornetzwerke aufgespannt. Hinter ambienten Systemen verbergen sich anspruchsvolle IT-Systeme, welche Fähigkeiten und Fertigkeiten des Menschen in Beruf, Alltag und Freizeit wirkungsvoll verstärken. Ambiente Systeme sind informationstechnische, verteilte, miniaturisierte Systeme, die über intelligente Sensorik und Aktorik verfügen. Gegenwärtig ist weltweit ein schneller Fortschritt zu verzeichnen, u.a. bedingt durch Entwicklungen im Bereich der technologischen Grundlagen sowie der fortschreitenden Miniaturisierung. Voraussichtlich werden ambiente Systeme erheblichen Anteil an der zukünftigen Wertschöpfung in den Industrienationen haben (siehe auch: Hightech-Strategie IKT 2020).

In der Medizin werden ambiente Systeme z. B. für die Anwendung in der Notfallerkennung und Rekonvaleszenz untersucht und entwickelt.

Weitere Informationen:

www.amsys-uni-kl.de

Filmtipp:

Interessierten Leserinnen und Lesern sei auch der Beitrag „Assisted Living“ des Fraunhofer-Instituts für Experimentelles Software Engineering empfohlen: http://www.youtube.com/ watch? $\mathrm{v}=\mid \mathrm{XH}$ sl6NZkbl

U. Kreimeier, München

\section{Deutsches Zentrum für Notfall- medizin und IT gegründet}

Informations- und Kommunikationssysteme spielen heute in der Rettungskette vom Notarzt bis zum Klinikum eine tragende Rolle. Zur effizienten notfallmedizinischen Versorgung der Bevölkerung ist deren zeit- und sachgemäße Optimierung notwendig.

Nur konsequent ist daher die Einrichtung des Deutschen Zentrums für Notfallmedizin und Informationstechnologie (DENIT) am Fraunhofer-Institut für Experimentelles Software Engineering (IESE) in Kaiserslautern. Medizinischer Leiter ist seit dem 1. Januar 2009 Dr. Thomas Luiz. Das DENIT widmet sich der Erforschung und Entwicklung von Systemen für optimierte Logistik und Kommunikation in der Notfallmedizin. Einsatzdatenbanken, Expertensysteme, Kommunikationsinfrastrukturen und telemedizinische Netzwerke sollen konzipiert und aufgebaut werden. Ziel ist es, die Erkenntnisse in die medizinische Praxis zu übertragen Darüber hinaus wird das Forschungszentrum umfangreiche Dienstleistungen für Industrie und öffentliche Hand erbringen. Hierzu zählen insbesondere der Technologietransfer auf dem Gebiet des Software und Systems Engineering, sowie die Entwicklung fortschrittlicher Schulungs- und Ausbildungsmaßnahmen (Simulation,

E-Learning) für das Rettungswesen.

Quelle:

Fraunhofer-Institut für Experimentelles Software Engineering IESE www.iese.fraunhofer.de 